\title{
ASOCIACIÓN ENTRE TUBERCULOSIS INFANTIL GRAVE E INMUNIZACIÓN PREVIA CON BCG EN UN HOSPITAL DE REFERENCIA NACIONAL, PERÚ 1990-2000
}

\author{
Félix Llanos-Tejada ${ }^{1,2, a}$, Hernán del Castillo ${ }^{3, a}$
}

\begin{abstract}
RESUMEN
El objetivo del estudio fue determinar la asociación entre la inmunización con BCG y tuberculosis grave (TB). Se realizó un estudio retrospectivo, se incluyó fichas de pacientes atendidos en el servicio de neumología del Instituto Nacional de Salud del Niño de Perú, entre los años 1990-2000. Se revisaron un total de 2106 casos de TB entre los que había 259 casos graves (TB miliar o meningoencefalitis por TB). Del total, 497 casos no tenían antecedente de inmunización con BCG; 202 tenían TB grave y 295 TB no grave (OR= 0,05; IC 95\%= 0,03-0,07). En conclusión, los niños con diagnóstico de TB y que han sido inmunizados con BCG, tienen $94 \%$ menos riesgo de desarrollar TB grave, en comparación a los niños con diagnóstico no inmunizados con BCG.
\end{abstract}

Palabras clave: Tuberculosis; Inmunización; BCG; Niño (fuente: DeCS BIREME).

\section{ASSOCIATION BETWEEN SEVERE TUBERCULOSIS IN CHILDREN AND PREVIOUS BCG IMMUNIZATION IN A NATIONAL REFERRAL HOSPITAL, PERU 1990-2000}

\begin{abstract}
ASBTRACT
The objective of the study was to determine the association between BCG immunization and severe tuberculosis (TB). We performed a retrospective study, including medical records from patients of the pneumology department at the National Children's Institute in Peru, between the years 1990-2000. A total of 2106 TB cases were reviewed, from them 259 patients were severe (miliary TB or meningoencephalitic TB). From all, 497 cases did not have history of BCG vaccination, 202 had severe TB and 295 non-severe TB $(\mathrm{OR}=0.05,95 \% \mathrm{Cl}=0.03$ to 0.07$)$. In conclusion, children diagnosed with TB and who have been immunized with BCG, has $94 \%$ lower risk of developing severe TB, compared to children with TB non-immunized with BCG.
\end{abstract}

Key words: Tuberculosis; Immunization; BCG; Child (source: MeSH NLM).

\section{INTRODUCCIÓN}

La tuberculosis (TB) continúa siendo un problema de salud pública pues cada minuto muere una persona de tuberculosis pulmonar en el mundo ${ }^{(1)}$. La historia de la "necesidad de detener" esta enfermedad se inicia desde los primeros trabajos de Koch, quien describe cepas de micobacterias atenuadas: la K1 de Koch, la cepa aviaria de Nocard, la BB de Burchard, Boez y Borrel, entre otros; pero, es finalmente Friedmann quien propone una vacuna preventiva y curativa. En 1921 los franceses Calmette y Guérin desarrollaron la cepa atenuada (a partir de la cepa de Nocard) que inició la inmunización contra TB en 120 recién nacidos a dosis de $2 \mathrm{mg}$ vía oral en tres dosis por dos semanas (2).

La vacunación con bacilo Calmette y Guérin (BCG) forma parte del Programa de Expansión de Inmunización de la OMS ${ }^{(1)}$. Sin embargo, en países occidentales la eficacia de la BCG no ha demostrado disminuir la tasa de incidencia o mortalidad por tuberculosis ${ }^{(3)}$. A pesar de esto, la inmunización con BCG es considerada un factor protector en la disminución de la incidencia y mortalidad de formas graves de TB, como son TB miliar y meningoencefalitis ${ }^{(4)}$. Existen estudios que prueban la utilidad de la inmunización con BCG en formas extrapulmonares de enfermedad, pero ninguno ha sido concluyente hasta el momento ${ }^{(4-6)}$.

A pesar de la existencia de varios metanálisis que muestran el efecto protector de la inmunización con BCG en las formas miliar y meníngea (formas graves de TB) y en la mortalidad por TB, estos no proveen datos necesarios para explorar la heterogeneidad de los estudios en el uso de cepas de BCG, administración de la vacuna y en el control de calidad de la cepa utilizada (7).

\footnotetext{
Servicio de Neumología, Hospital Nacional Dos de Mayo. Lima, Perú.

Servicio de Neumología, Clínica Vesalio. Lima, Perú.

Servicio de Neumología, Instituto Nacional de Salud del Niño. Lima, Perú.

Médico neumólogo
}

Recibido: 16-07-11 Aprobado: 25-01-12 
El impacto de la vacuna BCG sobre la epidemiología de la TB es escasa ya que no se ha demostrado protección contra las formas pulmonares (TBP) de la edad adulto, denominada TB secundaria; cuyos casos bacilíferos constituyen las principales fuentes de transmisión de la infección en la comunidad. Es necesario tener en cuenta que la protección demostrada de la vacunación se ejerce sobre la TB primaria, en niños menores de cinco años; cuyos casos prácticamente no contagian la enfermedad. La duración en tiempo, de esa protección sería aproximadamente de 10 años ${ }^{(5,6,8)}$.

El objetivo del presente estudio fue determinar el efecto protector de la inmunización con BCG sobre la TB infantil grave (TB miliar y meningoencefalitis TB); mediante la comparación de la frecuencia de casos graves entre niños atendidos en el Instituto Nacional de Salud del Niño (INSN) de Lima, Perú, con y sin antecedente de vacunación.

\section{EL ESTUDIO}

Se realizó un estudio tipo casos y controles de diseño analítico observacional. La muestra estuvo conformada por los pacientes atendidos en el programa de tuberculosis del Servicio de Neumología del Instituto Nacional de Salud del Niño (INSN).

Se procedió a la revisión de las fichas de atenciones del Programa de TB entre los años 1990 y 2000 . Estas fichas son formatos de atención anexas a las historias clínicas del establecimiento de salud.

Se denominó "casos" a los pacientes con forma grave de TB y "controles" a los pacientes con TB no-grave.

\section{DEFINICIÓN DE TÉRMINOS}

Tuberculosis (TB): enfermedad pulmonar o extrapulmonar demostrada por criterio clínico, radiológico o microbiológico (BK directo o cultivo) de acuerdo con los criterios de Stegen y Toledo, citados en la Norma Técnica de Control de TB del MINSA. En los casos de TB extrapulmonar el criterio clínico en este estudio incluirá la evaluación médica y de laboratorio que corresponda a cada entidad.

Inmunización con BCG: presencia de cicatriz en el hombro derecho o constancia de vacunación en la "Tarjeta de Crecimiento y Desarrollo de Niño Sano" de acuerdo con lo que se especificó en la ficha revisada.

TB Miliar: tuberculosis con confirmación radiológica de patrón miliar. No se incluyen las formas extrapulmonares sistémicas sin radiología compatible.
Meningoencefalitis TB (MEC-TB): tuberculosis con confirmación en LCR de cuadro compatible: pleocitosis a predominio de mononucleares, proteinorraquia $\mathrm{e}$ hipoglicorraquia. Puede ser con ADA positivo o negativo, según valor normal del laboratorio.

TB Grave: presencia de TB en las formas miliar o meníngea.

El estudio fue aprobado por el comité de investigación del Instituto Nacional de Salud del Niño.

Los datos se recogieron en el programa Excel 2005. Se realizó un análisis de riesgo calculando el Odds Ratio (OR), en el programa Epidat 3.1.

\section{HALLAZGOS}

En total, se obtuvieron 2106 casos de TB, de los cuales $1609(76,4 \%)$ correspondieron a TBP y $497(23,6 \%)$ a TB extrapulmonar sin compromiso pulmonar. En la Tabla 1 se resume el criterio utilizado para realizar el diagnóstico.

Se registraron 121 casos de TB miliar, lo que representó el $7,5 \%$ de los casos de TB pulmonar. Asimismo, se encontraron 138 casos de MEC-TB, representando el $27,8 \%$ de los casos de TB extrapulmonar.

La media de la edad fue de 67,2 $\pm 26,6$ meses, con rango de 13 días a 17 años. El sexo masculino fue el más frecuente con 1058 pacientes, lo que representó el $50,2 \%$ del total.

No hubo diferencias significativas entre los casos y controles para las variables demográficas intervinientes (estado nutricional, infección por $\mathrm{VIH}$ y enfermedades crónicas), que se muestran en la Tabla 2.

Del total, 259 casos fueron clasificados como TB grave (TB miliar o MEC-TB), lo que representa el $12,3 \%$ del total.

Se pudo determinar antecedente de inmunización con BCG en 1609 casos (76,4\%). La inmunización con BCG estuvo presente en 57 de 259 casos (22\%) de los pacientes con diagnóstico de TB grave y estaba presente en 1552 de 1847 casos (84\%) de los pacientes con TB no grave.

Al realizar el cálculo del riesgo, los niños que recibieron inmunización con BCG tenían un OR de 0,05 (IC 95\%= 0,03-0,07) veces para desarrollar formas graves de TB, en comparación a los que no fueron inmunizados. Esto se puede interpretar como que los niños con diagnóstico 
Tabla 1. Razones para el diagnóstico de tuberculosis pulmonar y extrapulmonar.

\begin{tabular}{lrr}
\hline & $\begin{array}{c}\text { TB pulmonar } \\
\mathbf{( \% )}\end{array}$ & $\begin{array}{c}\text { TB extra } \\
\text { pulmonar } \\
\text { (\%) }\end{array}$ \\
\hline TB criterio clínico & $150(9,3)$ & $419(84,3)$ \\
TB criterio microbiológico & $89(5,5)$ & $8(1,7)$ \\
TB criterio radiológico & $1370(85,2)$ & $70(14,8)$ \\
\hline
\end{tabular}

TB: tuberculosis.

de TB y que han sido inmunizados con BCG, tienen $94 \%$ menos riesgo de desarrollar TB grave, en comparación a los niños con diagnóstico no inmunizados con BCG.

\section{DISCUSIÓN}

La TB pulmonar es una enfermedad frecuente en nuestro medio, y la edad infantil es la definida como una población de riesgo que requiere estudios especiales para decidir la terapia. En edad pediátrica, esta enfermedad es frecuente en menores de 5 años, pero es menos frecuente entre los 5 a los 10 años de edad. En el presente estudio se ha encontrado que el promedio de edad es de 67,2 meses, edad que concuerda con lo descrito en otras series ${ }^{(1)}$.

La complejidad en el diagnóstico de TB en niños debido a la escasa proporción de hallazgos bacteriológicos y la utilización de los criterios de Stegen y Toledo, los que no siempre son certeros por lo que han sido cuestionados (1), hace que el presente estudio tenga como una limitación el poder determinar con exactitud la presencia de TB en la población evaluada. En la Tabla 1 se indica que el método para conseguir el diagnóstico en niños dependerá de la localización de la TB. Así, tenemos que en TB pulmonar el diagnóstico más frecuente se basa en el criterio radiológico, ya sea por radiografía de tórax o tomografía espiral multicorte con contraste de tórax; mientras el criterio bacteriológico es el menos frecuente por la dificultad reconocida de los pacientes pediátricos de poder expectorar. En los casos de TB extrapulmonar, podemos apreciar que el criterio clínico, que en el presente estudio ha considerado a la evaluación por médico especialista, es la forma de diagnóstico más frecuente.

Es interesante la alta frecuencia de TB miliar encontrada, aproximadamente el $7 \%$ de las formas de TB pulmonar y la alta frecuencia de MEC-TB, que equivale a un poco más de la cuarta parte de los casos de TB extrapulmonar. Informes previos mencionan una frecuencia de hasta $5 \%$ de las formas de TB grave, con una prevalencia
Tabla 2. Características demográficas de los niños con diagnóstico de tuberculosis grave o no grave en el Instituto Nacional de Salud del Niño, 1990-2000.

\begin{tabular}{lccc}
\hline & $\begin{array}{c}\text { TB grave } \\
(\mathbf{\%})\end{array}$ & $\begin{array}{c}\text { TB no grave } \\
\mathbf{( \% )}\end{array}$ & p \\
\hline Inmunización BCG & $57(22,0)$ & $1552(84,0)$ & $<0,005$ \\
\hline Procedencia de Lima & $155(59,8)$ & $1112(60,2)$ & 0,19 \\
\hline Edad en meses \pm DE & $67,3 \pm 13,7$ & $67,3 \pm 12,9$ & 0,23 \\
\hline Sexo & & & \\
$\quad$ Masculino & $131(50,5)$ & $930(50,4)$ & 0,55 \\
$\quad$ Femenino & $128(49,5)$ & $917(49,6)$ & 0,55 \\
Estado Nutricional & & & \\
\multicolumn{1}{c}{ Desnutrido } & $70(27,1)$ & $521(28,2)$ & 0,37 \\
$\quad$ Adecuado & $189(72,9)$ & $1326(71,8)$ & 0,29 \\
\hline Infección VIH & $7(2,7)$ & $56(3,0)$ & 0,32 \\
\hline Enfermedades crónicas & $15(5,7)$ & $112(6,1)$ & 0,43 \\
\hline Total & $\mathbf{2 5 9 ( 1 0 0 )}$ & $\mathbf{1 8 4 7}(\mathbf{1 0 0})$ & \\
\hline
\end{tabular}

DE: desviación estándar

menor al $1 \%$ en el rango de edad más frecuente de ocurrencia de TB infantil (1). Estos resultados se deban, quizás, a que el presente estudio ha sido realizado en el Servicio de Neumología del Instituto Nacional de Salud del Niño, centro de referencia a nivel nacional de casos de TB infantil, habiendo ocurrido un sesgo involuntario de selección.

La eficacia de la vacunación con el bacilo atenuado de Calmette y Güerin (BCG) es controversial, con una variación en la literatura que va del $0 \%$ a más del $80 \%{ }^{(4-7)}$. Es por esta inconsistencia en su eficacia que se están desarrollando nuevas terapias de prevención e inmunización alternativa a la BCG ${ }^{(9-11)}$. Aquellos que están a favor de la utilización de la BCG argumentan que esta vacuna previene la enfermedad pulmonar, la enfermedad meníngea y la enfermedad diseminada ${ }^{(5,7)}$.

Algunos autores proponen que el estado nutricional es un factor de riesgo para el desarrollo de TB, independiente del grado de severidad o gravedad; mientras otros refieren que el estado nutricional está en relación con la severidad o gravedad de la TBP; esto, debido a la respuesta inmunológica del hospedero ${ }^{(12,13)}$. En el presente estudio, el estado nutricional, la presencia de comorbilidad crónica y la presencia de infección por VIH no tienen relación con la presencia de TB grave.

Con respecto a la comorbilidad $\mathrm{TB}$ y $\mathrm{VIH}$, siempre se ha colocado en un apartado especial en las guías de tratamiento de TB (1), aunque Arbeláez et al. (14) no han podido encontrar una relación entre formas agresivas de TB e infección por $\mathrm{VIH}$, aun valorando el efecto protector de la BCG. Así, la OMS recomienda que se valore el riesgo y beneficio de la inmunización con 
BCG en pacientes con VIH, según la prevalencia de la enfermedad tuberculosa en el medio (1). En este estudio, tampoco se ha podido encontrar una relación existente entre la ocurrencia de TB grave en la población pediátrica con infección VIH evaluada.

El efecto protector de la inmunización con BCG encontrada en este estudio de una población pediátrica está de acuerdo con los estudios primarios de la inmunización (1-4). Aunque hay que considerar que el presente estudio es uno de los que ha demostrado un mayor efecto protector de la inmunización con BCG en la prevención de formas graves de tuberculosis de toda la literatura revisada.

La disminución del riesgo obtenida al recibir la vacunación, con un OR de 0,05, le da una alta potencia de validez, que por su diseño de corte retrospectivo, muestra un resultado alentador en protección de riesgo de desarrollar TB grave con la inmunización con BCG.

Minassian et al. (15) plantean la posibilidad de valorar consideraciones preclínicas antes de la aplicación de la inmunización con BCG, consideramos que este modelo no ayudaría en nuestro medio por el alto efecto protector encontrado en el presente estudio.

Asimismo, consideramos como un dato de suma importancia el hallazgo de que, a pesar de que la inmunización con BCG a todos los recién nacidos es obligatoria en nuestro medio, encontramos un porcentaje considerable de niños que no han sido inmunizados (casi el 25\%). Esta cifra, sin embargo, concuerda con la información que se encuentra en la página www.bcgatlas.org que confirma una cobertura de la vacunación con BCG en $80 \%$ en nuestro medio.

No ha sido motivo del presente estudio el valorar el efecto protector con respecto a la mortalidad, debido a que esos datos no se encuentran en todas las fichas revisadas, por cuanto un alto porcentaje de pacientes han continuado una terapia ambulatoria, que es lo que la Norma Técnica de Tratamiento del Control de la Tuberculosis estipula se realice nuestro medio.

Una limitación del presente estudio es el sesgo de selección de los datos recogidos. Las fichas de la Estrategia de TB del Instituto Nacional de Salud del Niño no fueron desarrolladas pensando en el presente estudio, de allí los problemas hallados en determinar ciertas variables como el nivel socioeconómico, procedencia de zona rural o urbana, y criterios diagnósticos utilizados. Se recomienda la realización de un estudio prospectivo con el desarrollo de una ficha que pueda recoger estos datos $\mathrm{u}$ otros que se consideren. Además, solo con un estudio longitudinal se podría determinar la verdadera asociación y el efecto protector de la inmunización de la BCG.

Otra limitación del presente estudio es el hecho de no poder valorar el impacto del nivel socioeconómico ni el tiempo de protección de la inmunización con BCG como variables confusoras. Estos datos no los hemos podido recoger de la ficha que se analizó para el presente estudio retrospectivo. Se recomienda un estudio prospectivo que pueda determinar el impacto estas variables. También recomendamos un estudio prospectivo que determine el tiempo de protección de la inmunización con BCG en nuestro medio y su impacto en zonas de alto riesgo y bajo riesgo de transmisión de TB.

En conclusión, un niño inmunizado con BCG tiene 94\% menos probabilidad (riesgo) de desarrollar un cuadro de TB grave en comparación a un niño no inmunizado con BCG.

\section{AGRADECIMIENTOS}

Al personal del Servicio de Neumología y del Programa de Control de Tuberculosis, del Instituto Nacional de Salud del Niño.

\section{Contribuciones de autoría}

Este fue un estudio donde el autor participó en la recolección y análisis de datos, el proceso de desarrollo del manuscrito y en la discusión del manuscrito.

\section{Fuentes de financiamiento}

Autofinanciado.

\section{Conflictos de interés}

El autor declara no tener conflictos de interés.

\section{REFERENCIAS BIBLIOGRÁFICAS}

1. World Health Organization. guidance for national tuberculosis programmes on the management of tuberculosis in children. Section 6: BCG vaccination in children. Geneva: WHO; 2006.

2. Yeager H. New drugs and vaccines. Dis Mon. 2007;53(1):59-63.

3. Teo SS, Shingadia DV. Does BCG have a role in tuberculosis control and prevention in the United Kingdom? Arch Dis Child. 2006;91(6):529-31.

4. Zhang LX, Tu DH, He GX, Ma ZQ, Nagelkerke NJ, Borgdorff $M W$, et al. Risk of tuberculosis infection and tuberculous meningitis after discontinuation of Bacillus CalmetteGuerin in Beijing. Am J Respir Crit Care Med. 2000;162(4 Pt 1):1314-7.

5. Aronson NE, Santosham M, Comstock GW, Howard RS, Moulton LH, Rhoades ER, et al. Long-term efficacy of BCG vaccine in American Indians and Alaska Natives: a 60-year follow-up study. JAMA. 2004;291(17):2086-91 
6. Boyd AE. BCG provides protection for a lifetime. Thorax. 2004;59:724.

7. Brewer TF. Preventing tuberculosis with bacillus CalmetteGuérin vaccine: a meta-analysis of the literature. Clin Infect Dis. 2000;31 Suppl 3:S64-7.

8. Sterne JA, Rodrigues LC, Guedes IN. Does the efficacy of BCG decline with time since vaccination? Int J Tuberc Lung Dis. 1998;2(3):200-7.

9. Doherty TM, Andersen P. Vaccines for tuberculosis: novel concepts and recent progress. Clin Microbiol Rev. 2005;18(4):687-702.

10. Martín C. The dream of a vaccine against tuberculosis; new vaccines improving or replacing BCG? Eur Respir J. 2005;26(1):162-7.

11. Vilaplana C, Gil O, Cáceres N, Pinto S, Díaz J, Cardona $P$. Prophylactic effect of a therapeutic vaccine against TB based on fragments of Mycobacterium tuberculosis. PLoS One. 2011;6(5):e20404.

12. Abubakar I, Matthews T, Harmer D, Okereke E, Crawford $\mathrm{K}$, Hall $\mathrm{T}$, et al. Assessing the effect of foreign travel and protection by BCG vaccination on the spread of tuberculosis in a low incidence country, United Kingdom, October 2008 to December 2009. Euro Survell. 2011;16(12):19826.
13. Guthmann JP, Antoine D, Fonteneau L, Che D, LévyBruhl D. Assessing BCG vaccination coverage and incidence of paediatric tuberculosis following two major changes in BCG vaccination policy in France. Euro Survell. 2011;16(12):19824.

14. Arbeláez MP, Nelson KE, Muñoz A. BCG vaccine effectiveness in preventing tuberculosis and its interaction with human immunodeficiency virus infection. Int $\mathrm{J}$ Epidemiol. 2000;29(6):1085-91.

15. Minassian AM, Ronan EO, Poyntz H, Hill AV, McShane $\mathrm{H}$. Preclinical development of an in vivo BCG challenge model for testing candidate TB vaccine efficacy. PLoS One. 2011;6(5):e19840.

Correspondencia: Félix Llanos-Tejada

Dirección: Av. Guardia Civil Sur 1053 B304, Lima 33, Perú.

Teléfono: (51) 99887731

Correo electrónico: neumofekollate@aol.com, felex3d@yahoo.com

\section{Consulte la versión electrónica de la} Revista Peruana de Medicina Experimental y Salud Pública en www.pubmed.gov

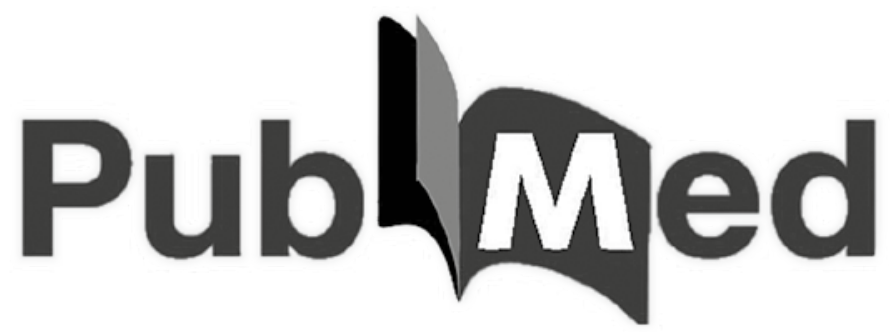

